\title{
NUEVOS DATOS SOBRE "LAS PORTADAS GÓTICAS GADITANAS": EL PATROCINIO DEL CARDENAL DIEGO HURTADO DE MENDOZA EN LA PARROQUIA DE SANTIAGO DE JEREZ Y LA AUTORÍA DE RODRIGO DE ALCALÁ EN LA PARROQUIA DE SAN JORGE DE ALCALÁ DE LOS GAZULES
} \author{
DE ALCALÁ DE LOS GAZULES \\ David Caramazana Malia \\ andreacidm@gmail.com \\ Manuel Romero Bejarano \\ hout.mg@hotmail.com
}

NEW CONTRIBUTIONS TO "LAS PORTADAS GÓTICAS GADITANAS": THE SPONSORCHIP OF THE CARDINAL DIEGO HURTADO DE MENDOZA IN THE CHURCH OF SANTIAGO DE JEREZ AND THE AUTHORSHIP OF RODRIGO DE ALCALÁ IN THE CHURCH OF SAN JORGE

Entre las últimas décadas del siglo XV y comienzos del siglo XVI se hicieron en Jerez y su entorno una serie de portadas inspiradas en las laterales de la cabecera de la Catedral de Sevilla. De entre todas, este artículo destaca la de la epístola de la jerezana parroquia de Santiago y la principal de la iglesia de San Jorge de Alcalá de los Gazules. La primera por ser realizada bajo el patrocinio del cardenal Hurtado de Mendoza y la segunda por estar firmada por el maestro constructor, algo en lo que hasta el momento nadie había reparado.

Palabras clave: Diego Hurtado de Mendoza, Rodrigo de Alcalá, gótico, Jerez de la Frontera y Alcalá de los Gazules. 
Between the last decades of the XV century and the beginning of the XVI, in Jerez and its environment there were made a series of façades inspired in the lateral ones from the head of the Seville Cathedral. Amongst them, this article emphasizes the Epistle Façade of the Church of Santiago de Jerez, and the main one from the Church of San Jorge, located in Alcalá de los Gazules of San Jorge. The first one is important because of its execution under the sponsorship of the Cardinal Hurtado de Mendoza, and the second one for presenting its builder master's signature, a fact that never has been pointed out until now.

Keywords: Diego Hurtado de Mendoza, Rodrigo de Alcalá, gothic, Jerez de la Frontera and Alcalá de los Gazules.

En 1993 Carlos García Peña publicaba "Portadas góticas gaditanas" en el que establecía una clasificación tipológica de varias portadas ubicadas en templos de la actual provincia de Cádiz. En el artículo se establecen dos grupos, las más antiguas son de estilo mudéjar, y las más recientes derivan de las laterales de la cabecera de la Catedral de Sevilla, que son de las que vamos a tratar en este artículo ${ }^{1}$.

\section{LA PORTADA DE LA EPÍSTOLA DE LA PARROQUIA DE SANTIAGO DE JEREZ DE LA FRONTERA}

La iglesia de Santiago de Jerez de la Frontera nació como una pequeña ermita construida al exterior de una de las puertas del recinto amurallado de origen almohade. El crecimiento de la población a lo largo del siglo XIV hizo que al final de la centuria el templo ya hubiese sido convertido en parroquia para dar servicio al arrabal surgido en aquel sector ${ }^{2}$. A fines del siglo XV el primitivo edificio era insuficiente para el número de feligreses, por lo que se emprendió una reconstrucción. No se conocen muchos datos documentados de la nueva obra, si bien esta hubo de comenzarse poco antes de diciembre de 1496, pues por esa fecha se estaba delimitando el solar en el que habría de levantarse la fábrica ${ }^{3}$. La unidad estilística, un tanto alterada por las intervenciones de época contemporánea ${ }^{4}$ y los

${ }^{1}$ GARCÍA PEÑA, Carlos: "Portadas góticas gaditanas", en Revista Goya, nº 198. Madrid, 1987, pp. 326-331.

2 JIMÉNEZ LÓPEZ DE EGUILETA, Javier E.: "La iglesia en Jerez durante el siglo XIV”, en JIMÉNEZ LÓPEZ DE EGUILETA, Javier E. y POMAR RODIL, Pablo J.: Limes Fidei. 750 años de cristianismo en Jerez, Jerez de la Frontera, 2014, pp. 101-113.

${ }^{3}$ ROMERO BEJARANO, Manuel: "Del mudéjar al gótico. Arquitectura religiosa a finales del XV en Jerez", en BAREA RODRÍGUEZ, Manuel A. y ROMERO BEJARANO, Manuel: Actas del congreso conmemorativo del 750 aniversario de la incorporación de Jerez a la Corona de Castilla 1264-2014, Jerez de la Frontera, 2015, pp. 437-458.

4 ÁlVAREZ LUNA, Ángeles, GUERRERO VEGA, José María y ROMERO BEJARANO, Manuel: La intervención en el patrimonio. El caso de las iglesias jerezanas (18502000), Jerez de la Frontera, 2003, pp.75-100. 
problemas estructurales cíclicos que ha sufrido ${ }^{5}$, hacen pensar en una construcción rápida, de a lo sumo veinticinco años, algo poco común para un conjunto de las dimensiones del que se trata. La observación detenida del mismo nos sirve para corroborar esta teoría, ya que la portada de la epístola ostenta el escudo (Figura 1) del cardenal Diego Hurtado de Mendoza, arzobispo de Sevilla entre 1485 y 1502, así que ha de ser anterior a esta última fecha, y por tanto su construcción hubo de ser de los primeros elementos de la nueva iglesia.

Diego Hurtado de Mendoza y Quiñones ${ }^{6}$ perteneció al influyente linaje de los Mendoza de Guadalajara. Sobrino del célebre arzobispo de Toledo Pedro Hurtado de Mendoza y hermano de Íñigo López de Mendoza, conde de la Tendilla, su período como arzobispo de Sevilla transcurrió en su mayor parte en la corte de los Reyes Católicos. Hasta el momento, la única obra que se adscribía a su patrocinio era la Capilla de la Antigua de la Catedral de Sevilla ${ }^{7}$, construida para su enterramiento, y aun así, Recio Mir piensa que fue una decisión sobrevenida, ya que aspiraba a la mitra de Toledo, que al final fue asignada a Gonzalo Jiménez

${ }^{5}$ ÁlVAREZ LUNA, Ángeles, GUERRERO VEGA, José María y ROMERO BEJARANO, Manuel: "Los problemas estructurales de la parroquia de Santiago de Jerez de la Frontera: "Los sistemas de construcción aplicados a la restauración monumental", en $A c$ tas del IV Congreso Nacional de Historia de la Construcción, Madrid, 2004, pp. 35-47. En la actualidad la iglesia de Santiago se encuentra cerrada y en obras para tratar de subsanar sus eternos problemas estructurales.

${ }^{6}$ ALONSO MORGADO, José: Prelados sevillanos o episcopologio de la Santa Iglesia Metropolitana y Patriarcal de Sevilla, Sevilla, 1906, pp.386-393; LAYNA SERRANO, Francisco: Historia de Guadalajara y sus Mendozas en los siglos XV y XVI, Madrid, 1993, vol. 2, pp. 237-240.

${ }^{7}$ Sobre la capilla de la Antigua, véase ALONSO RUIZ, Begoña: "El laboratorio arquitectónico. La huella de la catedral de Sevilla en la arquitectura religiosa del tardogótico", en JIMÉNEZ MARTÍN, Alfonso (ed.): La Piedra Postrera. V Centenario de la conclusión de la catedral de Sevilla, Sevilla, 2007, pp. 260-261; NUSSBAUM, Norbert: "Space and form redefined. Paradigm shifts in german architecture, 1350-1550", en JIMÉNEZ MARTÍN, Alfonso (ed.): La Piedra Postrera. V Centenario de la conclusión de la catedral de Sevilla, Sevilla, 2007, tomo I, pp. 326-327; JIMÉNEZ MARTÍN, Alfonso: "Rarezas de la capilla de la Antigua de la Catedral de Sevilla", en JIMÉNEZ MARTÍN, Alfonso (ed.): La Piedra Postrera. V Centenario de la conclusión de la catedral de Sevilla, Sevilla, 2007, tomo II, pp. 401-420; JIMÉNEZ MARTÍN, Alfonso: Anatomía de la Catedral de Sevilla, Sevilla, 2013, pp. 184 y ss. PINTO PUERTO, Francisco y ANGULO FORNOS, Roque: "Decisiones constructivas en la ejecución de la Capilla de La Antigua de la catedral de Sevilla. Estudio a través de modelos gráficos", en HUERTA FERNÁNDEZ, Santiago y FUENTES GONZÁLEZ, Paula: Actas del Noveno Congreso Nacional y Primer Congreso Internacional Hispanoamericano de Historia de la Construcción, Madrid, 2015, pp. 1337-1347. 
de Cisneros ${ }^{8}$. En el caso de las obras diocesanas la portada que nos ocupa sería la única obra costeada bajo su patrocinio, porque hasta el momento que sepamos, no se ha encontrado su escudo en ningún otro edificio del antiguo Arzobispado Hispalense. Con todo, la inclusión del blasón en la portada de Santiago es bastante discreta, hasta el punto de que ningún investigador que se ha ocupado de la puerta había reparado en el mismo ${ }^{9}$. Se podría plantear aquí que la inclusión en la portada de Santiago del blasón del prelado podría indicar tan sólo su adscripción al periodo cronológico en que se realizó la obra, como sucedió en otras prelaturas posteriores como las de los hermanos Teodoli en Cádiz, cuyo escudo se puede ver en las parroquias de Santa María la Coronada de Medina Sidonia o El Divino Salvador de Vejer, cuando consta que ambas fueron costeadas por sus respectivas fábricas con la ayuda del duque de Medina Sidonia ${ }^{10}$. En tal caso, el escudo de Hurtado de Mendoza, y el de sus sucesores, figuraría en otras portadas parroquiales de la época, sin ir más lejos las otras dos de Santiago, la principal de San Mateo de Jerez o la principal de Santa María de Arcos de la Frontera, por sólo poner algunos ejemplos. Con todo, no se han podido localizar apuntes contables que corroboren esta teoría.

La portada de la epístola de Santiago (Figura 2) se desarrolla entre dos contrafuertes de planta romboidal decorados en el primer cuerpo por repisas y

${ }^{8}$ RECIO MIR, Álvaro: "La llegada del Renacimiento a Sevilla: El proyecto del Cardenal Hurtado de Mendoza para la Capilla de la Antigua de la Catedral", en Archivo Español de Arte, nº 290, 2000, pp. 182-190.; SÁNCHEZ HERRERO, José: "Sevilla del Renacimiento", en ROS, Carlos: Historia de la Iglesia en Sevilla, Sevilla, 1992, pp. 311-312.

${ }^{9}$ Sería prolijo referir aquí todos los trabajos que tratan sobre la construcción de la iglesia de Santiago por lo que sólo citaremos los más destacados: GRANDALLANA Y ZAPATA, Luis de: Noticia histórico-artística de algunos de los principales monumentos de Jerez, Jerez de la Frontera, 1885, pp. 29 y ss.; ROMERO DE TORRES, Enrique: Catálogo monumental de España. Provincia de Cádiz (1908-1909), Madrid, 1934, pp. 420-441; SANCHO DE SOPRANIS, Hipólito: Introducción al estudio de la arquitectura en Jerez, Jerez de la Frontera, 1934, pp. 9 y ss.; ESTEVE GUERRERO, Manuel: Jerez. Guía oficial de Arte, Jerez de la Frontera, 1952, pp. 152 y ss.; GARCÍA PEÑA, Carlos: "Portadas góticas gaditanas", en Revista Goya, n 198, Madrid, 1987, pp. 326-331; Ib: Arquitectura gótica religiosa en la Provincia de Cádiz. Diócesis de Jerez, Madrid, 1990, pp. 644 y ss.; POMAR RODIL, Pablo J. y MARISCAL RODRÍGUEZ, Miguel Ángel: Jerez. Guía artística y monumental, Madrid, 2004, pp. 155 y ss.; ROMERO MEDINA, Raúl y ROMERO BEJARANO, Manuel: "Un lugar llamado Jerez. El maestro Alonso Rodríguez y sus vínculos familiares y profesionales en el contexto de la arquitectura del tardogótico en Jerez de la Frontera", en JIMÉNEZ MARTÍN, Alfonso (Ed.): La Catedral después de Carlín. Actas de la XVII Aula Hernán Ruiz, Sevilla, 2010, pp. 175-288; ROMERO BEJARANO, Manuel: "Del mudéjar al gótico...", op. cit., p. 446 y ss.

${ }^{10}$ ROMERO BEJARANO, Manuel: Maestros y obras de ascendencia portuguesa en el tardogótico de la Baja Andalucía, Tesis doctoral inédita. Juan Rufo Teodoli ocupó la sede gaditana entre 1523 y 1525 y su hermano Jerónimo entre este último año y 1565. 
doseletes, y en el segundo por pináculos ornamentados con crochets. El vano es adintelado, estando coronado por un arco apuntado con tres arquivoltas y trasdosado por un gablete que presenta en su exterior cardinas y un florón como coronamiento. En las enjutas, a modo de tapiz, hayamos dos cuerpos superpuestos de tracería ciega. La portada responde al segundo grupo de Portadas góticas gaditanas. Este es el que deriva de las portadas laterales de la cabecera de la Catedral de Sevilla y comprende un conjunto de obras realizadas en un período dilatado de tiempo, el cual abarca al menos entre 1484, fecha que aparece en la inscripción conmemorativa de la portada del evangelio de la parroquia jerezana de San Miguel $^{11}$, y hacia 1524, fecha en que hubo de realizarse la portada principal de la parroquia de San Jorge de Alcalá de los Gazules, un caso un tanto atípico del que nos ocuparemos al final.

Además de la diferencia cronológica, encontramos otra muy clara en lo referido a la ornamentación escultórica, pues se pueden establecer dos grupos bien diferenciados: las que carecen de escultura figurativa y las que la poseen. En el conjunto de las portadas menos ornamentadas se encuentran la de la epístola de la parroquia de Nuestra Señora de la O de Chipiona, las laterales de San Miguel, la del evangelio de Santiago y la principal de San Mateo, estas tres últimas en Jerez de la Frontera. En el segundo grupo además de la que nos ocupa están la principal del Monasterio de la Victoria, la principal (que quedó inacabada) de la Prioral de El Puerto de Santa María, la principal de la propia parroquia de Santiago de Jerez y la ya citada de Alcalá de los Gazules. Todas las del segundo grupo presentan un bestiario y una especial profusión de elementos ornamentales, como tracerías y baquetones

Con todo, la de la epístola de Santiago es la más ornamentada de todas, pues incluye una figura en el tímpano (sólo presente en Alcalá de los Gazules) y dos figuras bajo los doseletes de los estribos en las laterales, algo que ninguna de las otras posee, quizá porque se perdieron o con más probabilidad porque nunca se hicieron. Estas esculturas de bulto redondo colocadas bajo sus respectivas hornacinas se relacionan con la catedral de Sevilla. En las jambas de Santiago podemos ver bajo dosel a un lado al arcángel San Gabriel (Figura 3) y al otro la Virgen María (Figura 4) conformando la escena de la Anunciación; en la hornacina del tímpano la figura de Santiago Apóstol (Figura 5). Las figuras de la Anunciación,

${ }^{11}$ GRANDALLANA Y ZAPATA, Luis de: Noticia histórico-artística de algunos de los principales monumentos de Jerez, Jerez, 1885, p. 21. En honor a la verdad hay que decir que el primero que hace mención a esta lápida, sin llegar a publicarla completa, fue Bartolomé Gutiérrez (GUTIÉRREZ, Bartolomé: Historia y Anales de la muy noble y muy leal Ciudad de Jerez de la Frontera. Jerez, 1887, Tomo II, libro III, p. 141) quien al tratar del año 1482 nos dice que "Este año se dio principio a la puerta de S. Miguel que mira al Arenal y duro dos años hacer la solo ella; así lo dice una lápida que tiene sobre el diente interior donde asegura haverse empezado año 1482 y acabado año 1484”. 
pese al deterioro de la piedra, presentan una calidad muy superior a las figuras del bestiario que a continuación citaremos. El tratamiento de los paños es mucho más delicado, manteniendo influencias con el norte de Europa, y las proporciones de la figura se acercan más a los cánones clásicos. El ángel aparece con la rodilla izquierda levemente adelantada en señal de reverencia, sin que sepamos cual era la disposición de sus brazos, pues los ha perdido. La Virgen, que tampoco tiene brazos, permanece firme de pie en una postura muy estatuaria. Un detalle a tener en cuenta es el pie derecho de San Gabriel, cuyos dedos se doblan noventa grados hasta apoyar en el basamento de la hornacina, este rasgo se observa en ciertas figuras de la portada del Nacimiento de la Catedral de Sevilla, en concreto con las obras del taller de Pedro Millán, de un calidad estética inferior a las que están firmadas por el maestro, lo que podría darnos pistas sobre su autoría ${ }^{12}$. Respecto al Santiago de la hornacina del tímpano se trata de una escultura de pequeño tamaño realizada en barro cocido y tal vez en origen policromado. El santo aparece de pie con la mano derecha adelantada, con la cual hubo de sostener el bastón de peregrino, mientras que en la izquierda se observa un libro. El tratamiento de los paños y las facciones del santo, recuerdan también al entorno de Pedro Millán, no obstante la ubicación de la pieza dificulta un análisis más pormenorizado.

Aunque el tiempo ha causado estragos en la piedra, podemos reconocer algunas de las figuras del bestiario de esta portada que se distribuyen entre las arquivoltas y el dintel de la puerta. Aquí identificamos ángeles orantes y peregrinos, lo que cuadra perfectamente con el santo titular representado en el tímpano. También son identificables San Cristóbal y Sansón (Figura 6), así como dos figuras desnudas en las jambas que bien podrían representar a Adán y Eva. Dentro de una de las arquivoltas encontramos otra figura de mujer desnuda, pero en esta ocasión muestra claramente su sexo. Este motivo iconográfico (quizás la lujuria) no es extraño a la arquitectura del último gótico de Jerez, pues aparece en una de las ménsulas del claustro principal del convento de Santo Domingo, en la portada de acceso al claustro grande en la cartuja de Santa María de la Defensión e incluso el motivo se adentra en el renacimiento pues se nos muestra en la portada de la capilla de los Siles en la iglesia conventual de San Francisco. En el campo de los animales (reales o fantásticos) la portada de Santiago permite identificar dos dragones, un cocodrilo como encarnación del mal, dos monos, un águila y un cordero, figuras estas últimas que por su ubicación a los pies de Santiago podrían representar a San Juan y Jesucristo respectivamente. Pasando al estilo de estas figuras, hay que señalar que están tratadas con gran tosquedad y en muchos casos su tallado es esquemático. La desproporción de los miembros es evidente, con un cuerpo muy pequeño y una cabeza muy grande en la que se

${ }^{12}$ LAGUNA PAÚL, Teresa: "Las portadas del Bautismo y del Nacimiento de la Catedral de Sevilla", en Bienes culturales: revista del Instituto del Patrimonio Histórico Espanol, $N^{o} 1$, Madrid, 2002, pp. 83-100. 
remarcan los ojos. Sus formas permiten relacionarlas con otras esculturas ornamentales de diferentes edificios de Jerez, que pudieron ser realizados por el mismo taller. En este caso se encuentran los ángeles orantes hallados en el arco de la primitiva puerta principal de la parroquia de San Marcos, realizados en torno a 1515, y los tenantes del arco de acceso al claustro grande de la Cartuja de Santa María de la Defensión fechados entre 1523 y $1525^{13}$.

Volviendo con la erección de la portada de Santiago, hay que señalar que fue uno de los primeros elementos del templo en estar terminado, siendo un caso similar al del Monasterio de la Victoria de El Puerto de Santa María ${ }^{14}$. En ambos casos el afán del comitente era que su patrocinio quedase bien claro. No por casualidad Hurtado de Mendoza elige la portada de la epístola del templo, cuando lo lógico hubiese sido la principal. Sin embargo el proceso constructivo de la parroquia fue un tanto extraño, ya que para respetar la orientación hacia el este del presbiterio hubo que encajar la fachada principal entre el caserío. No sucedía así con la de la epístola, que quedaba abierta a un gran espacio dispuesto delante de una de las puertas de la muralla de la ciudad, con lo que de facto se convertía en la portada principal, o al menos la más visible, algo que aún hoy en día es así. Además cuenta con la ventaja de ser de menores dimensiones que la principal y por tanto más fácilmente realizable para un prelado que veía que avanzaba su episcopado sin dejar su huella en la archidiócesis. Con todo, resulta curiosa la discreción con que se incluye en la obra el escudo del arzobispo, pues se halla en un pequeño azulejo dentro del tímpano al lado de la imagen del titular del templo, en lugar de incluir un gran escudo de piedra coronando el gablete, como el de la portada principal de esta iglesia, que ostenta el blasón de los Reyes Católicos, o el de la Victoria de El Puerto, que hace lo propio con el de los duques de Medinaceli.

\section{LA PORTADA PRINCIPAL DE LA PARROQUIA DE SAN JORGE DE ALCALÁ DE LOS GAZULES. RODRIGO DE ALCALÁ Y SU POSIBLE INTERVENCIÓN EN LA PORTADA DE LA PARROQUIA DE SANTA MARÍA DE ARCOS DE LA FRONTERA}

La historia de la parroquia de San Jorge de Alcalá de los Gazules se nos presenta un tanto difusa. Se cree que en su emplazamiento existió en tiempos de la dominación islámica una mezquita que, tras la conquista cristiana, fue transformada en iglesia parroquial, junto a otras dos de la población. En 1524 el marqués de Tarifa, señor del lugar, consiguió una bula de Clemente VII para la reunificación en San Jorge de las tres parroquias, momento en el que se piensa que se inició

${ }^{13}$ ROMERO BEJARANO, Manuel: Maestros y obras..., op. cit.

${ }^{14}$ ROMERO MEDINA, Raúl: "Los canteros de la obra tardogótica del Monasterio de la Victoria de El Puerto de Santa María”, en Revista de Historia de El Puerto, El Puerto de Santa María, 2010, nº 44, pp. 59-78. 
una reconstrucción integral del edificio. No obstante, el grueso de la fábrica corresponde al siglo XVII ${ }^{15}$, si bien se conserva un elemento que puede datarse en las primeras décadas del XVI, que no es otro que la portada principal.

Esta portada ha sido analizada por varios autores ${ }^{16}$, si bien ninguno de ellos ha acertado a ver una inscripción, clara y meridiana, que hay en la parte inferior del tímpano, y dice:

\section{$\mathrm{R}^{\circ} \mathrm{D}^{\prime} \mathrm{ALCALA}$}

Con lo que nos encontramos con una obra firmada por un maestro tardogótico de la zona jerezano-gaditana hasta ahora poco conocido. Rodrigo de Alcalá está documentado en Jerez de la Frontera entre 1515 y $1544^{17}$. Sabemos de su intervención en la obra de la capilla mayor de la parroquia de Santa María de la Oliva de Lebrija ${ }^{18}$, si bien su participación fue testimonial pues estuvo trabajando aquí un mes y casi a punto de finalizar la edificación. Por otra parte Rodrigo de Alcalá contrató en 1527 la obra del dormitorio bajo del monasterio de Santo Domingo de Jerez en unas condiciones muy ventajosas, lo que hace pensar que por esas fechas era un maestro de mucho prestigio ${ }^{19}$. Tanto en Lebrija como en Jerez aparecen claras referencias al último gótico portugués, y de hecho está documentada en su cuadrilla la presencia de canteros provenientes de ese país.

En cuanto a la cronología, la fecha post quem de 1524 propuesta, encaja a la perfección con el desarrollo profesional de Rodrigo de Alcalá, que podría haber realizado la portada de Alcalá de los Gazules antes de intervenir en el jerezano convento de Santo Domingo o incluso simultanear ambas obras, como era frecuente en los constructores de la época. La portada (Figura 7) se enmarca en la tradición de portadas góticas derivadas de las laterales de la cabecera de la

15 AA.VV.: Guía Artística de Cádiz y su provincia, Sevilla, 2005, Tomo II, pp. 221 y ss.

${ }^{16}$ En orden cronológico, los despistados historiadores han sido los siguientes ROMERO DE TORRES, Enrique: Catálogo monumental de España..., op. cit., p. 441; RAMOS ROMERO, Marcos: Alcalá de los Gazules, Cádiz, 1983, pp. 311 y ss.; ARANDA LINARES, Carmen y ALONSO DE LA SIERRA FERNÁNDEZ, Lorenzo: "Arte medieval”, en FLORES FERNÁNDEZ, Ana María (coord.): Cádiz y su provincia, Sevilla, 1984, pp. 175-201; GARCÍA PEÑA, Carlos: "Portadas góticas...”, op. cit., pp. 326-331; Ibídem, p. 221.

${ }^{17}$ ROMERO BEJARANO, Manuel: Maestros y obras..., op. cit.

18 ROMERO MEDINA, Raúl y ROMERO BEJARANO, Manuel: "La Catedral de Sevilla y su conexión con la arquitectura del tardogótico portugués", en JIMÉNEZ MARTÍN, Alfonso (Ed.): La Catedral entre 1434-1517: Historia y conservación, Actas de las XX edición del Aula Hernán Ruiz, Sevilla, 2013, pp. 237-276.

19 JIMÉNEZ LÓPEZ DE EGUILETA, Javier E. y ROMERO BEJARANO, Manuel: Los claustros de Santo Domingo de Jerez de la Frontera, Jerez de la Frontera, 2013, pp. 56 y ss. 
Catedral de Sevilla. Al igual que estas y otros de sus correlatos en la zona gaditana, el vano rectangular se encuentra inserto en un arco apuntado con varias arquivoltas decoradas con figuras de bestiario coronado por un gablete, presenta tracería ciega en la parte superior y queda enmarcado por dos estribos de planta romboidal rematados por pináculos decorados con crochets. Sin embargo, en Alcalá de los Gazules, se observa una evolución del modelo. En los lados exteriores de los estribos se pueden apreciar estructuras cilíndricas anilladas de claro aspecto columnario similares a las que hay en el exterior de la capilla del Rosario de la parroquia de Santa María de Arcos de la Frontera o en el cuerpo superior de la portada del palacio del Conde del Águila de la misma población. En Jerez los encontramos en la portada principal de la parroquia de San Marcos, donde intervino Diego Ximénez de Alcalá, quizás hermano de Rodrigo de Alcalá ${ }^{20}$. Por su parte los estribos romboidales que flanquean al vano carecen de baquetones, siendo éstos sustituidos por estructuras prismáticas giradas que crean el mismo efecto de claroscuro, en esto encontramos también similitud con la parroquia de Santa María de Arcos de la Frontera pues el mismo efecto se repite en la fachada principal, donde también los estribos presentan complejas divisiones horizontales formadas por molduras cuadrangulares giradas y colocadas a diferentes alturas. En Alcalá, la tracería ciega de la zona superior nada más que alcanza a la altura del gablete, mientras que más arriba el paramento queda liso y tan solo decorado con dos ventanas ciegas a los lados. Se trata de vanos apuntados ornamentados con tracería en la zona superior.

Quizás lo más llamativo de esta portada sea la actualización de la decoración del tímpano, frente a la hornacina coronada con dosel de algunas de las portadas del entorno jerezano, Rodrigo de Alcalá imita lo que en esos años estaba haciendo Miguel Perrín en las portadas de la Catedral de Sevilla ${ }^{21}$, pues ocupa todo el espacio disponible en el tímpano con un relieve que representa a San Jorge liberando a la princesa Sabra del dragón, bordeado por una banda de caireles (Figura 8). El santo, de una escala desproporcionada, aparece montado a caballo, con una espada en el cinto y pese a lo peligroso de su misión, va vestido a la moda del momento y en la cabeza lleva un complicado sombrero adornado con una gigantesca pluma, si bien en el pecho y las rodillas muestra piezas de armadura. El santo lancea a un pequeño dragón, representado con una ingenuidad deliciosa. Tras él, la princesa aparece ataviada a la moda con un vestido de mangas acuchilladas y un sombrero. Luce una larga melena y está arrodillada y con las manos unidas, en actitud de súplica. Sin ser una pieza escultórica de primera calidad, el

${ }^{20}$ ROMERO BEJARANO, Manuel: Maestros y obras..., op. cit.

${ }^{21}$ LAGUNA PAÚL, Teresa: "Cultura visual y promoción artística del escultor Miguel Perrin en la Catedral de Sevilla (1517-1552)", en LUGAND, Julien (ed.): Échanges artistiques entre la France et l'Espagne ( $S$. XV fin XIX siecles), Universidad de Perpignan, 2012, pp. 143-163. 
tratamiento de las figuras puede considerarse correcto, por lo que estamos ante otro caso de arquitecto escultor, como sucede con Fernando Álvarez o Pedro Fernández de la Zarza ${ }^{22}$. De que la ejecución material del relieve es obra de Rodrigo de Alcalá no debe quedarnos duda, pues su firma aparece en el mismo bloque de piedra en que está realizado.

Por cronología la de Alcalá de los Gazules sería una de las últimas portadas góticas gaditanas, si bien la principal de la Prioral de El Puerto de Santa María (Figura 9), aun estando inacabada, presenta en varios puntos las citadas estructuras de molduras cuadrangulares giradas, y en la parte exterior de los estribos unas curiosas estructuras cilíndricas superpuestas de aspecto columnario adornadas con baquetones, lo que supone una evolución importante con respecto a los modelos más antiguos y que la relacionan con la portada de Alcalá. Según los últimos estudios la obra de El Puerto quedó interrumpida en 1512, precisamente por la portada que nos ocupa ${ }^{23}$, pero en la obra de la portada se han detectado tres fases diferentes ${ }^{24}$ que en algunos casos interfieren con elementos anteriores; por lo que la cronología tal vez debería de ser revisada a la vista del descubrimiento de la firma de Rodrigo de Alcalá.

El epílogo llega con la fachada principal de la parroquia de Santa María de Arcos de la Frontera (Figura 10). La similitud con Alcalá de los Gazules es evidente, como demuestran las molduras cuadrangulares giradas y las estructuras cilíndricas de los laterales, pero también es clara su relación con la obra de Juan Gil de Hontañón, en especial con la fachada principal de la Catedral de Salamanca. Este hecho ya fue señalado por García Peña ${ }^{25}$, y nosotros estamos en pleno acuerdo con él. La obra de la iglesia de Santa María de Arcos se inicia en torno a 1520 y muy probablemente lo más antiguo sea la portada principal, para la que bien pudo dar una traza Juan Gil de Hontañón. No obstante, el primer proyecto fue modificado, podríamos decir que enriquecido, pues los estribos laterales ocultan elementos ornamentales de la zona más antigua, algo a todas luces impensable si se tratase de un diseño unitario. En este caso están las molduras curvas que adornan las esquinas superiores del primer cuerpo (muy similares a

${ }^{22}$ ROMERO BEJARANO, Manuel y ROMERO MEDINA, Raúl: "Pedro Fernández de la Zarza: un maestro tardogótico de la Baja Andalucía (1494-1569)", en ALONSO RUIZ, Begoña (ed.): La Arquitectura tardogótica castellana entre Europa y América, Madrid, 2011, pp. 197-212.

${ }^{23}$ ROMERO MEDINA, Raúl: "Fábrica, obra y mecenazgo arquitectónico: los Medinaceli y la Prioral de El Puerto de Santa María entre Juan de Hoces y Alonso Rodríguez (1478-1512)", en GONZÁLEZ GÓMEZ, Juan Miguel y MEJÍAS ÁLVAREZ, María Jesús (Eds.): Estudios de Historia del Arte. Centenario del Laboratorio de Arte (1907-2007), Sevilla, 2009, Tomo II, pp. 401-415.

${ }^{24}$ RUIZ DE LA ROSA et alii: “La Prioral de El Puerto de Santa María. El proyecto gótico original”, Sevilla, 2010, pp. 56 y ss.

${ }^{25}$ GARCÍA PEÑA, Carlos: Arquitectura gótica religiosa..., op. cit., pp. 121 y ss. 
las que se pueden ver en la portada principal de la iglesia matriz de Vila Nova de Foz Coa, en Portugal) prácticamente invisibles por los estribos que la flanquean, por tanto es muy posible que el propio Rodrigo de Alcalá, en cuya obra es constante la presencia de elementos del último gótico portugués, modificase el trazado inicial de la obra.

En comparación con otros maestros constructores del momento, han sido pocas las obras que se han podido documentar de Rodrigo de Alcalá. De hecho, tan sólo queda clara su intervención en el dormitorio del monasterio de Santo Domingo de Jerez y la fachada de la parroquia de Alcalá de los Gazules. No obstante, las ventajosas condiciones en las que contrata la obra jerezana, hacen pensar que en 1527 ya era un maestro de prestigio en el ámbito local. Si se confirmase con documentos su intervención en la parroquia de Santa María de Arcos estaríamos ante un maestro de primera fila. Es de esperar que futuras investigaciones puedan ampliar la nómina de edificios trazados y construidos por este artífice.

Fecha de recepción: 15 de septiembre de 2015

Fecha de aceptación: 20 de noviembre de 2015 


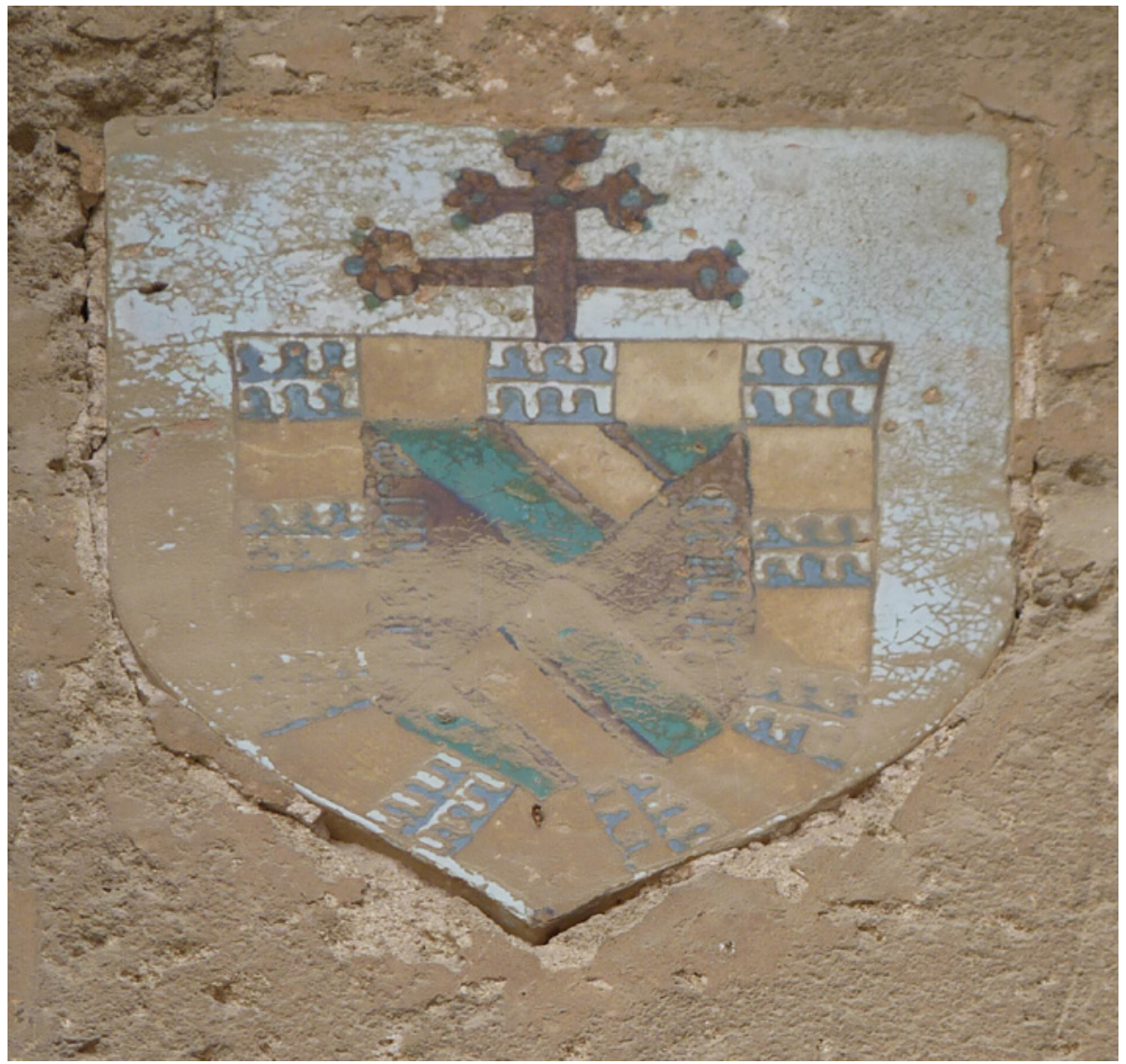

Figura 1. Parroquia de Santiago de Jerez de la Frontera, fachada de la epístola, detalle del escudo del cardenal Diego Hurtado de Mendoza, c. 1502. 
Figura 2. Parroquia de Santiago de Jerez de la Frontera, fachada de la epístola, vista general, c. 1502. 


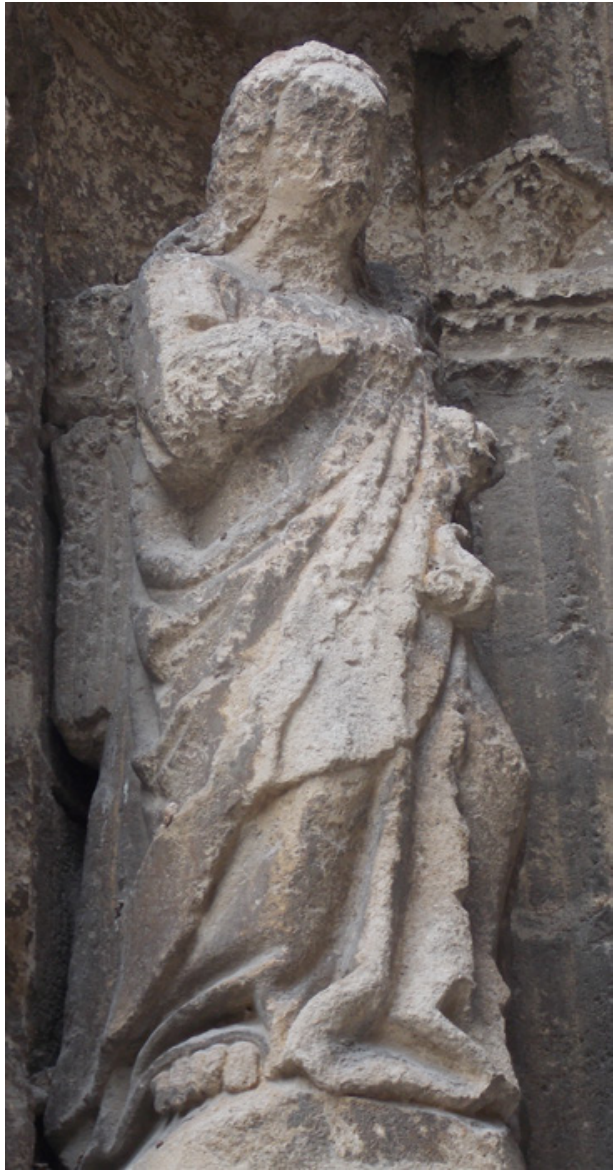

Figura 3. Parroquia de Santiago de Jerez de la Frontera, fachada de la epístola, escultura del Arcángel San Gabriel, taller de Pedro Millán, c. 1502.

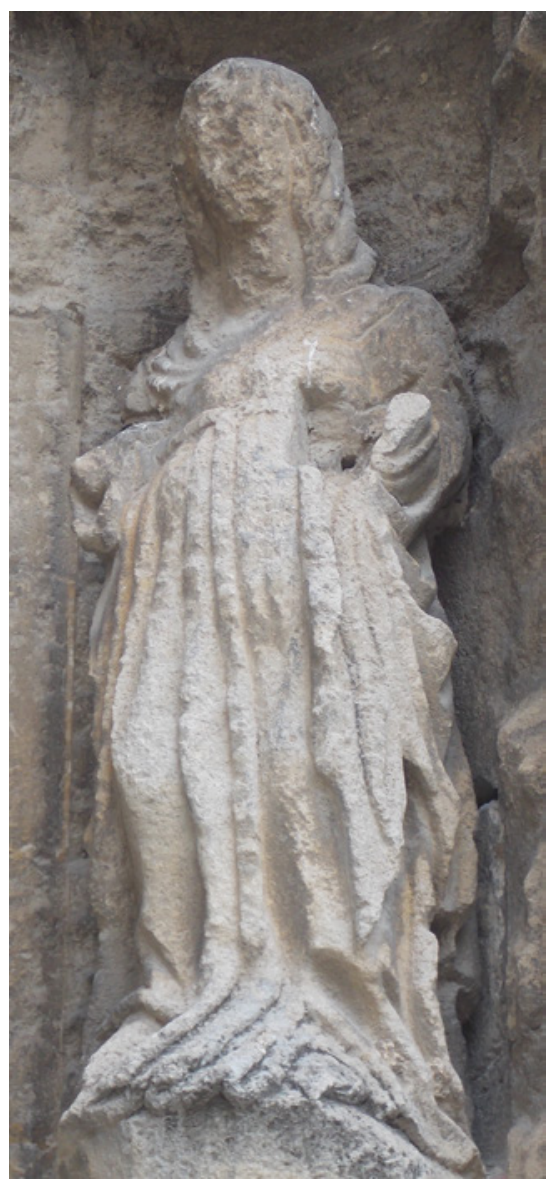

Figura 4. Parroquia de Santiago de Jerez de la Frontera, fachada de la epístola, escultura de la Virgen María, taller de Pedro Millán, c. 1502. 


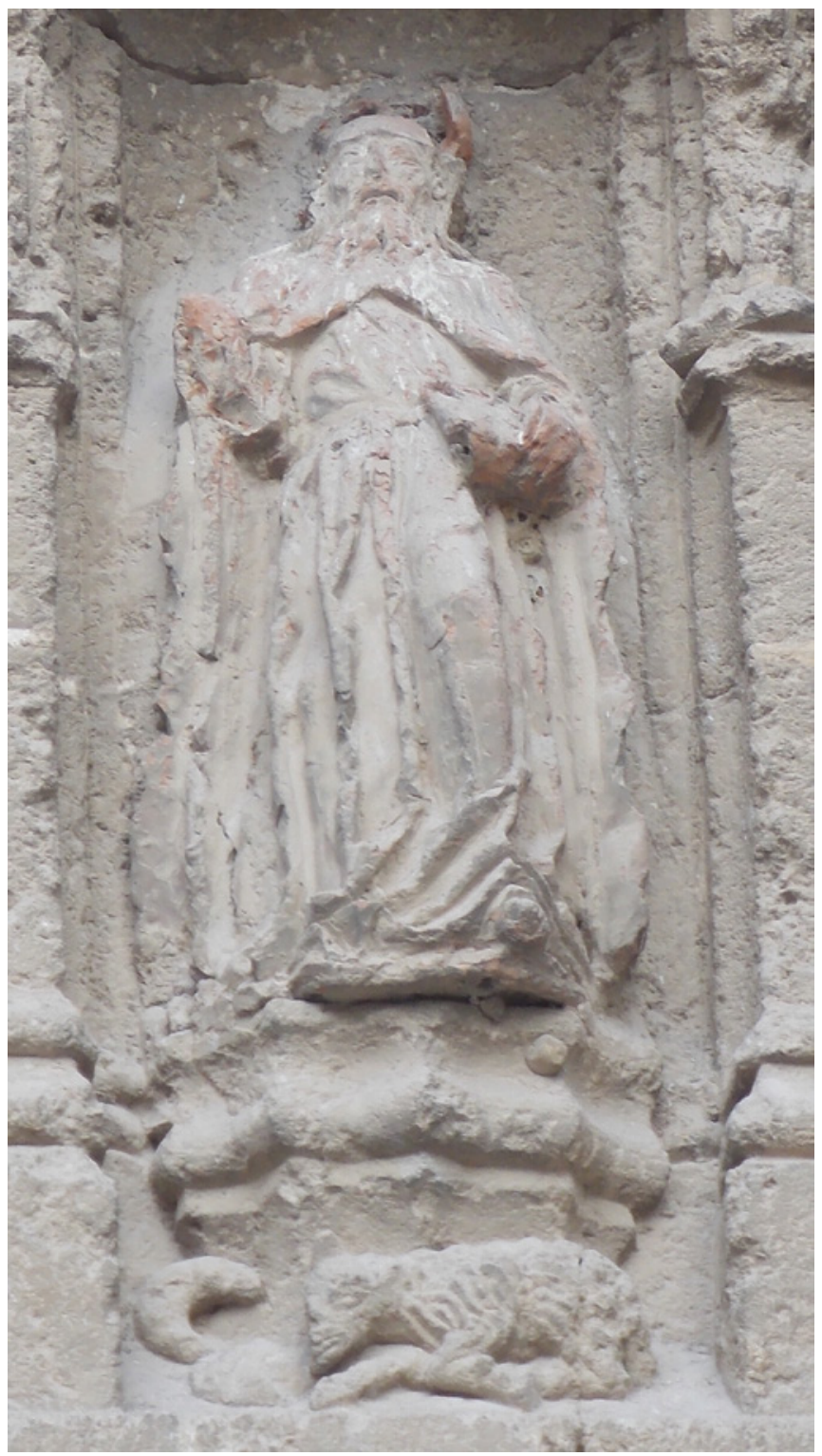

Figura 5. Parroquia de Santiago de Jerez de la Frontera, fachada de la epístola, escultura de Santiago, taller de Pedro Millán, c. 1502 . 


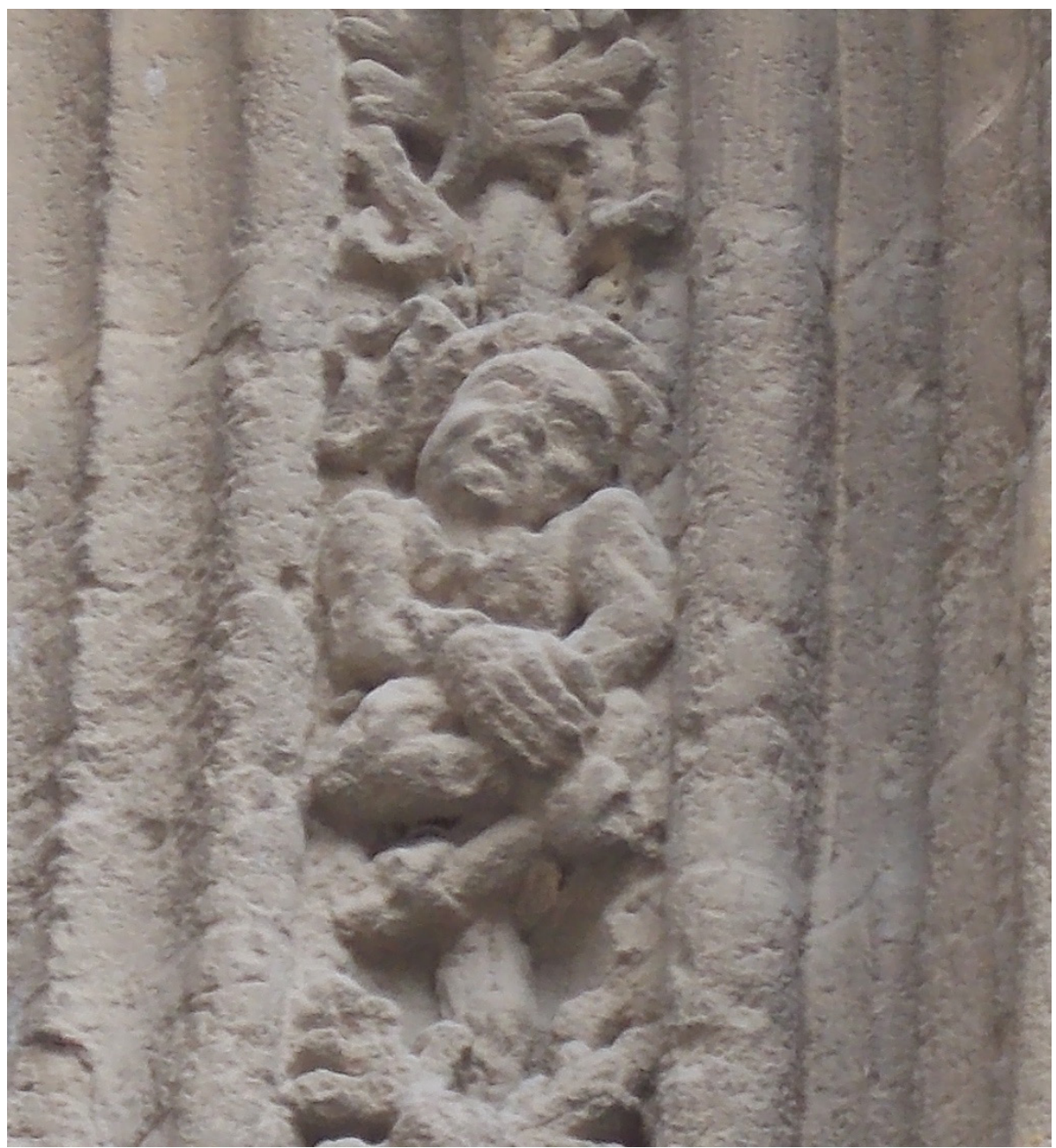

Figura 6. Parroquia de Santiago de Jerez de la Frontera, fachada de la epístola, detalle del bestiario, c. 1502. 


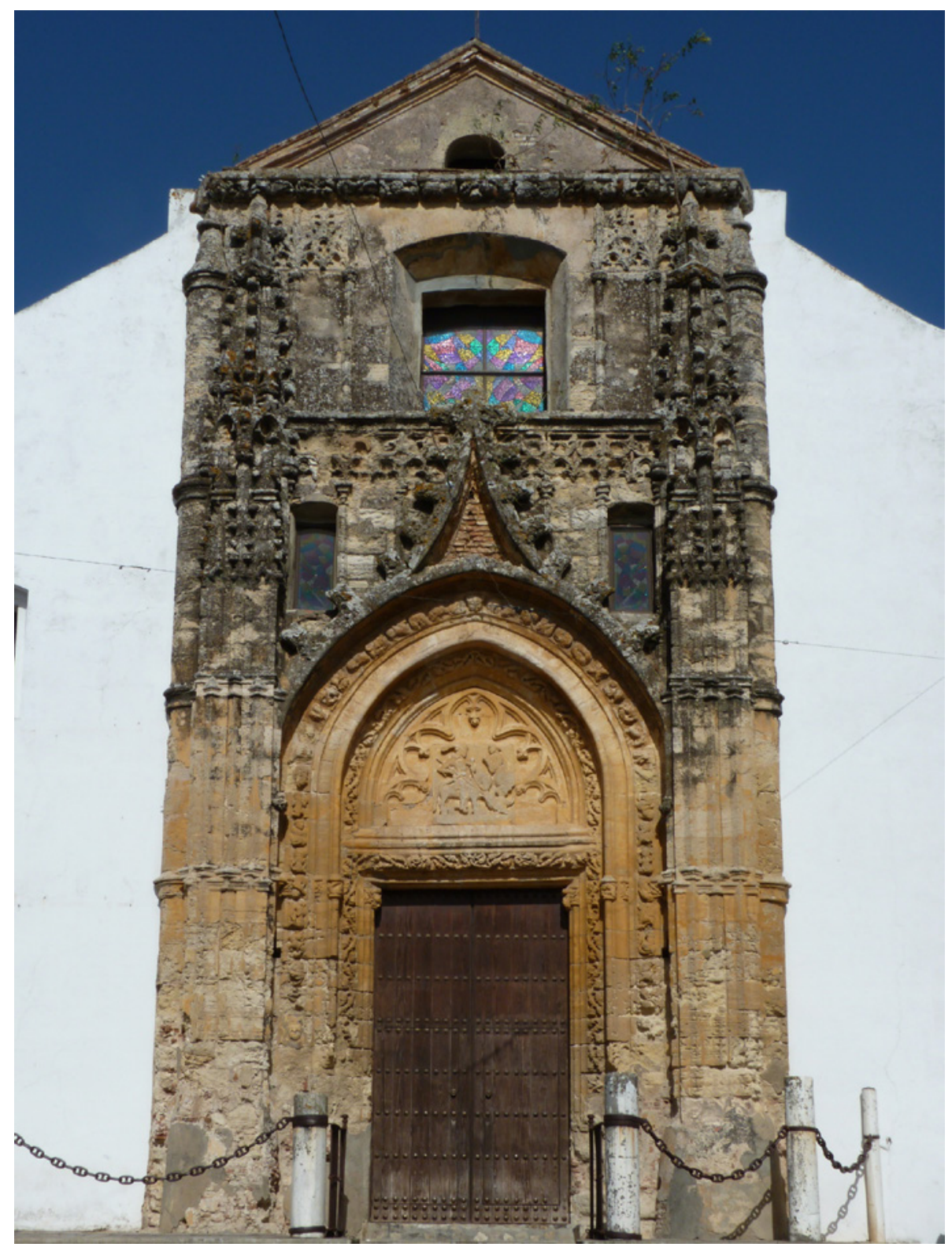

Figura 7. Parroquia de San Jorge de Alcalá de los Gazules, fachada principal, Rodrigo de Alcalá, c. 1524. 


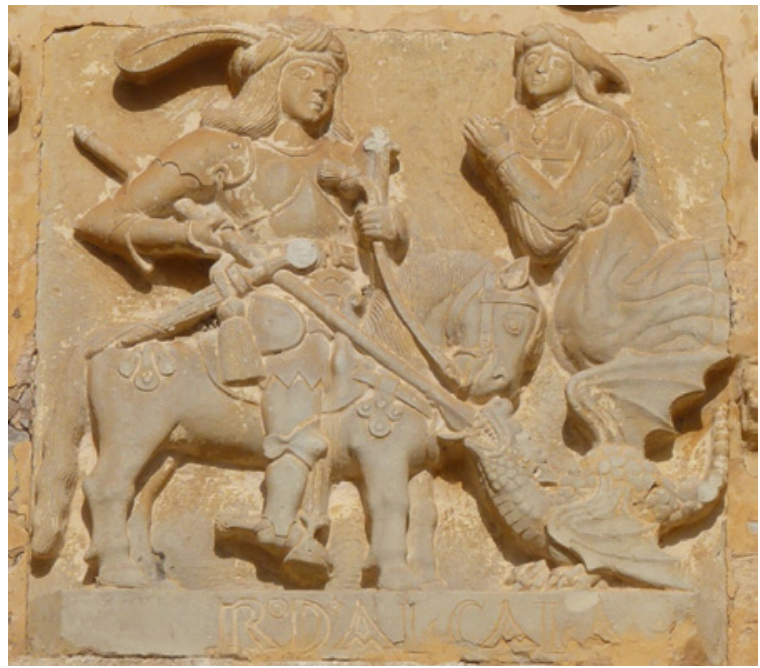

Figura 8. Parroquia de San Jorge de Alcalá de los Gazules, fachada principal, detalle del tímpano con la firma de Rodrigo de Alcalá, c. 1524.

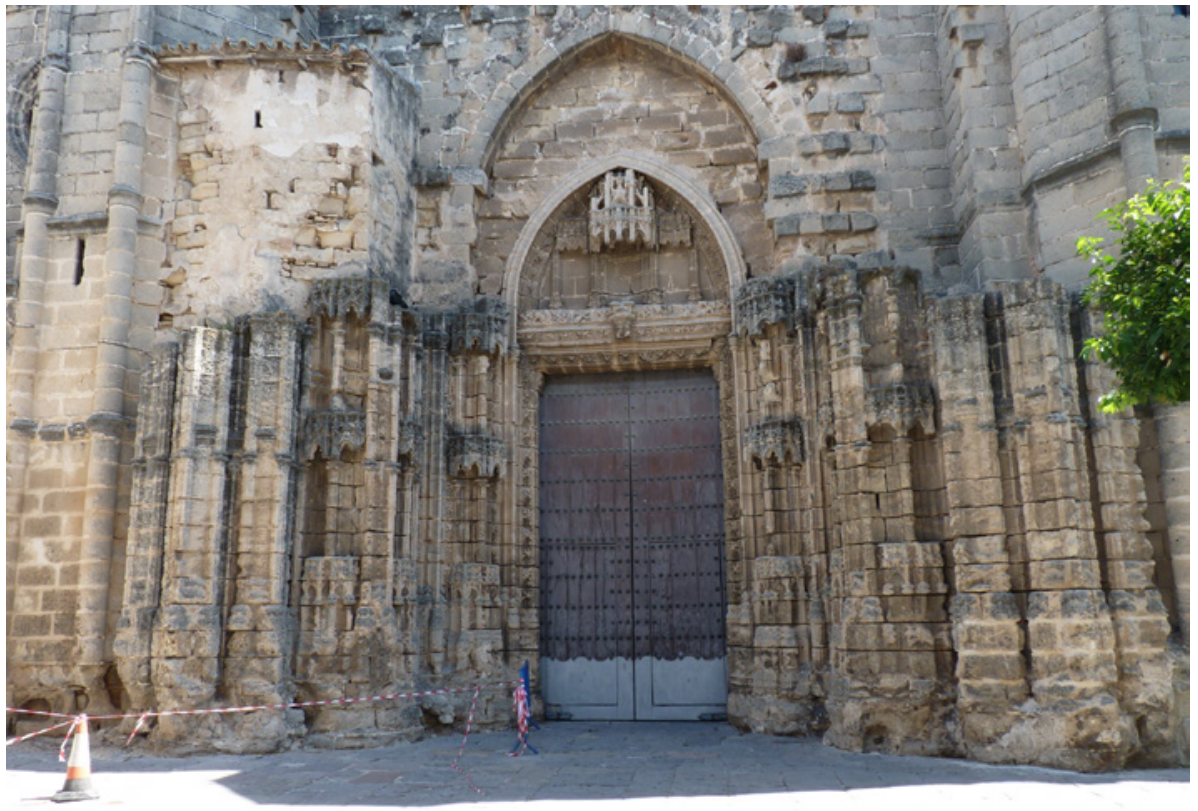

Figura 9. Iglesia Prioral de El Puerto de Santa María, fachada de los pies, c. 1512. 


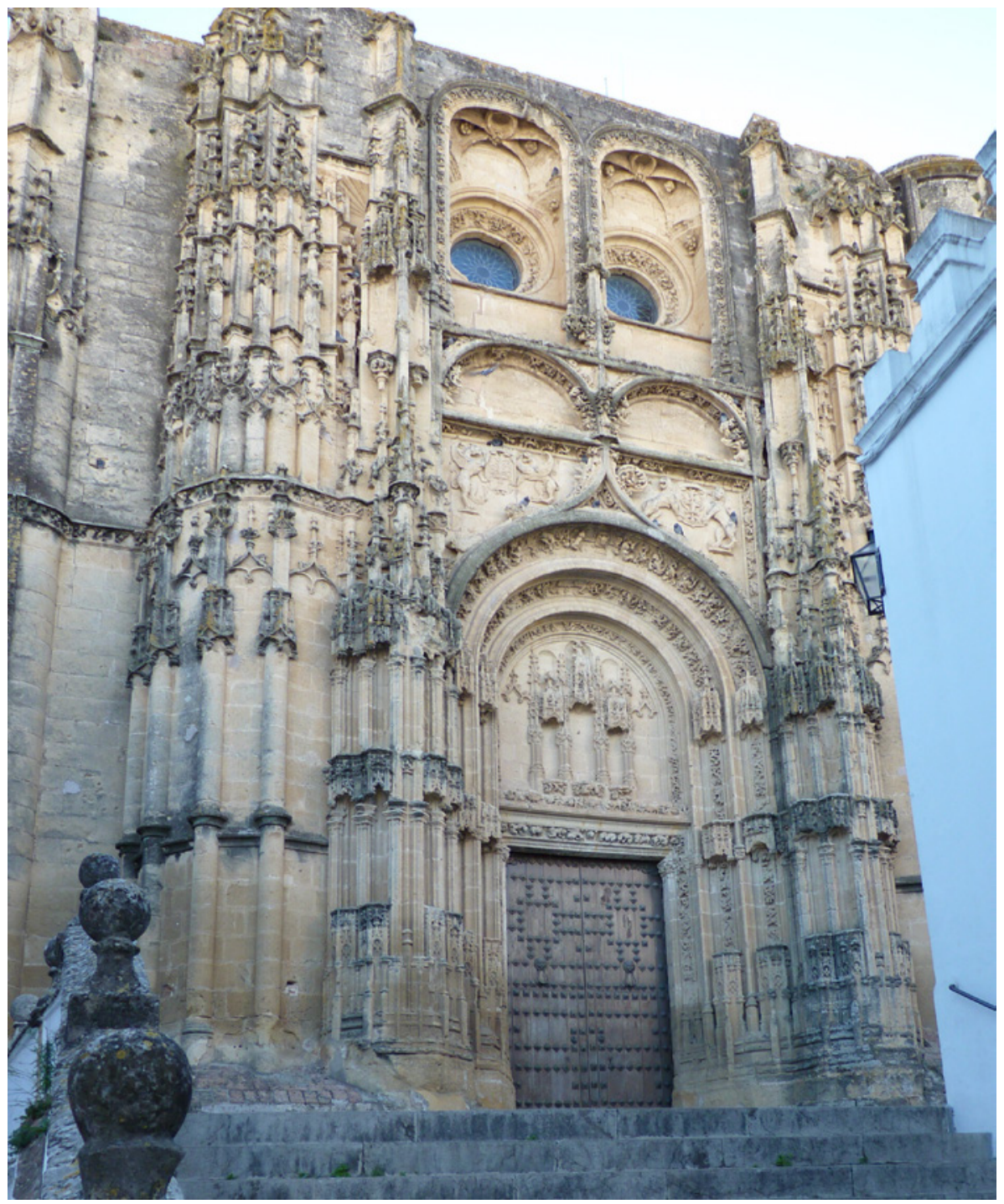

Figura 10. Parroquia de Santa María de Arcos de la Frontera, fachada principal, c. 1525. 